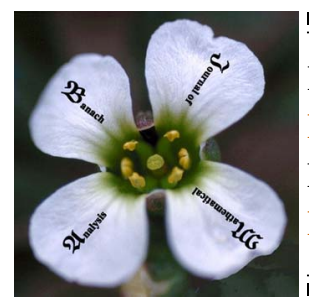

Banach J. Math. Anal. 10 (2016), no. 1, 15-26

http://dx.doi.org/10.1215/17358787-3158354

ISSN: $1735-8787$ (electronic)

http://projecteuclid.org/bjma

\title{
THE STRONG GELFAND-PHILLIPS PROPERTY IN BANACH LATTICES
}

\author{
HALIMEH ARDAKANI, ${ }^{*}$ S. MOHAMMAD MOSHTAGHIOUN, \\ S. M. SADEGH MODARRES MOSADEGH, and MANIJEH SALIMI
}

Communicated by D. E. Alspach

\begin{abstract}
We introduce the concept of the strong Gelfand-Phillips (GP) property in Banach lattices, and we characterize Banach lattices with the strong GP property. Next, by introducing the class of almost limited completely continuous operators from an arbitrary Banach lattice $E$ to another $F$, we give some properties of them related to some well-known classes of operators and, especially, to the strong GP property of the Banach lattice $E$.
\end{abstract}

\section{INTRODUCTION AND PRELIMINARIES}

A subset $A$ of a Banach space $X$ is called limited if every weak* null sequence $\left(x_{n}^{*}\right)$ in $X^{*}$ converges uniformly on $A$; that is,

$$
\lim _{n \rightarrow \infty} \sup _{a \in A}\left|\left\langle a, x_{n}^{*}\right\rangle\right|=0 .
$$

Also, a subset $A$ of a Banach lattice $E$ is called almost limited (see [6]) if every disjoint weak* null sequence $\left(x_{n}^{*}\right)$ in $E^{*}$ converges uniformly on $A$.

Every relatively compact subset of $E$ is limited and so it is almost limited. If every limited subset of a Banach space $X$ is relatively compact, then $X$ has the Gelfand-Phillips (GP) property. For example, the classical Banach spaces $c_{0}$

Copyright 2016 by the Tusi Mathematical Research Group.

Received Dec. 6, 2014; Accepted Mar. 25, 2015.

${ }^{*}$ Corresponding author.

2010 Mathematics Subject Classification. Primary 46B42; Secondary 46B50, 47B65.

Keywords. almost limited set, limited completely continuous operator, Gelfand-Phillips property. 
and $\ell_{1}$ have the GP property, and every separable Banach space, every Schur space (i.e., where the weak and norm convergence of sequences in $X$ coincide), spaces with their duals containing no copy of $\ell_{1}$, such as reflexive spaces, and $C(K)$-spaces, where $K$ is both compact and sequentially compact, have the same property (see [4]). Also, we recall that a Banach space $X$ has the GP property if and only if every limited and weakly null sequence $\left(x_{n}\right)$ in $X$ is norm null (see $[7])$. The reader can find some useful and additional properties of limited sets and Banach spaces with the GP property in [7] and [9].

In this article, we introduce an alternative form of the GP property for Banach lattices. In fact, a Banach lattice $E$ has the strong GP property if every almost limited set in $E$ is relatively compact. Then, in Section 3, by introducing the concept of almost limited completely continuous (alcc) operators between Banach lattices, we obtain some characterizations of them and then we treat the relation between the strong GP property of $E$ and alcc operators on $E$.

Here, we remember some definitions and terminologies from Banach lattice theory. It is evident that, if $E$ is a Banach lattice, then its dual $E^{*}$, endowed with the dual norm and pointwise order, is also a Banach lattice. The norm $\|\cdot\|$ of a Banach lattice $E$ is order-continuous if, for each generalized net $\left(x_{\alpha}\right)$ such that $x_{\alpha} \downarrow 0$ in $E,\left(x_{\alpha}\right)$ converges to 0 for the norm $\|\cdot\|$, where the notation $x_{\alpha} \downarrow 0$ means that the net $\left(x_{\alpha}\right)$ is decreasing, its infimum exists, and $\inf \left(x_{\alpha}\right)=0$. A Banach lattice $E$ is said to be $\sigma$-Dedekind complete if every countable subset of $E$ that is bounded above has a supremum. A subset $A$ of $E$ is called solid if $|x| \leq|y|$ for some $y \in A$ implies that $x \in A$ and the solid hull of $A$ is the smallest solid set containing $A$ and it is exactly the set $\operatorname{Sol}(A)=\{y \in E:|y| \leq|x|$, for some $x \in A\}$.

Throughout this article, $X$ and $Y$ denote the arbitrary Banach spaces, and $X^{*}$ refers to the dual of the Banach space $X$. We use $L_{w^{*}}\left(X^{*}, Y\right)$ for Banach spaces of all bounded weak*-weak continuous operators from $X^{*}$ to $Y$. Also $E, F$, and $G$ denote arbitrary Banach lattices; $E^{+}=\{x \in E: x \geq 0\}$ refers to the positive cone of the Banach lattice $E$; and $B_{E}$ is the closed unit ball of $E$.

If $x$ is an element of a Banach lattice $E$, then the positive part, negative part, and absolute value of $x$ are denoted by $x^{+}, x^{-}$, and $|x|$, respectively. If $a, b$ belong to $E$ and $a \leq b$, then the interval $[a, b]$ is the set of all $x \in E$ such that $a \leq x \leq b$. A subset of a Banach lattice is called order-bounded if it is contained in an order interval. A linear mapping $T$ from $E$ into $F$ is called order-bounded if it carries order-bounded subsets of $E$ into order-bounded sets. The lattice operations in the dual Banach lattice $E^{*}$ are weak* sequentially continuous if, for every weak* null sequence $\left(f_{n}\right)$ in $E^{*},\left|f_{n}\right| \rightarrow 0$ for $\sigma\left(E^{*}, E\right)$. For undefined terminology, we refer the reader to the classical references [2], [1], and [11].

\section{The strong Gelfand-Phillips property in Banach lattices}

As we said before, a Banach lattice $E$ has the strong GP property if all almost limited subsets of $E$ are relatively compact. It is clear that the strong GP property implies the GP property. Also, the strong GP property is inherited by closed sublattices. In the following we characterize some classes of Banach lattices with 
the strong GP property. At first, we recall from [11] that an element $x$ belonging to a Riesz space $E$ is discrete if $x>0$ and $|y| \leq x$ implies $y=t x$ for some real number $t$. If every order interval $[0, y]$ in $E$ contains a discrete element, then $E$ is said to be a discrete Riesz space.

Theorem 2.1. Let $E$ be a discrete Banach lattice with order-continuous norm. Then E has the strong GP property.

Proof. Let $A$ be an almost limited subset of $E$, and let $B=\operatorname{Sol}(A)$ be the solid hull of $A$. By order continuity of the norm on $E$ and [6, Remark 2.7], $B$ is almost limited. Since $E$ is a discrete Banach lattice with order-continuous norm by [11], the lattice operations in $E^{*}$ are weak* sequentially continuous, and, by [10, Theorem 2.6], $B$ is limited. Since $E$ has an order-continuous norm, $E$ has the GP property by [15, Theorem 4.5], and $B=\operatorname{Sol}(A)$ (and hence $A$ itself) is relatively compact. Therefore $E$ has the strong GP property.

The classical Banach lattices $c_{0}, \ell_{p}$, where $1 \leq p<\infty$, and Schur spaces are discrete with order-continuous norm and so they have the strong GP property.

Remember that a Banach lattice is an $A M$-space if $x \wedge y=0$ implies $\|x \vee y\|=$ $\max \{\|x\|,\|y\|\}$.

Corollary 2.2. Let $E$ be an $A M$-space with order-continuous norm; then $E$ has the strong GP property.

Proof. Note first that if $E$ is an $A M$-space with an order-continuous norm, then $E$ is discrete (see [15, proof of Theorem 1.4]), and Theorem 2.1 now shows that $E$ has the strong GP property.

Theorem 2.3. Let $E$ be a $\sigma$-Dedekind complete Banach lattice. Then the following are equivalent:

(a) E is discrete with order-continuous norm;

(b) E has the strong GP property.

Proof. (a) $\Rightarrow$ (b). It follows from Theorem 2.1.

$(\mathrm{b}) \Rightarrow(\mathrm{a})$. By definition of almost limitedness, every order interval in a Banach lattice is almost limited if and only if the sequence $\left(\left|f_{n}\right|\right)$ is weak* null for every disjoint weak* null sequence $\left(f_{n}\right)$ in $E^{*}$. Then by [6, Lemma 2.2], in the $\sigma$-Dedekind complete Banach lattice $E$, every order interval is an almost limited set, and because of the hypothesis, it is compact. Now by [1, Corollary 21.13], $E$ is discrete with order-continuous norm.

However, the following example shows that $\sigma$-Dedekind completeness of the theorem cannot be removed. In fact, a discrete Banach lattice $c$ of all convergent sequence of scalars is not $\sigma$-Dedekind complete, and so its norm is not ordercontinuous, but it has the strong GP property.

Theorem 2.4. The Banach lattice $c$ has the strong GP property.

Proof. If $c$ does not have the strong GP property, then there exists an almost limited sequence $\left(y_{n}\right)$ in $c$ which has no convergent subsequence. Each $y_{n}$ has a representation $y_{n}=\left(y_{n, k}\right)_{k=1}^{\infty}$, where $y_{n, k}$ converges to $\alpha_{n}$ as $k \rightarrow \infty$. The 
boundedness of $y_{n}$ implies that the sequence $\left(\alpha_{n}\right)$ is bounded and so it has a convergent subsequence. Without loss of generality, we may assume that $\left(\alpha_{n}\right)$ is convergent. Let $x_{n}=\left(y_{n, k}-\alpha_{n}\right)_{k=1}^{\infty}$. Then each $x_{n}$ belongs to $c_{0}$. For each $m \neq n$,

$$
\left\|x_{n}-x_{m}\right\| \geq\left\|y_{n}-y_{m}\right\|-\left\|\alpha_{n}-\alpha_{m}\right\|
$$

and the convergence of the sequence $\left(\alpha_{n}\right)$ implies that the sequence $\left(x_{n}\right)$ has no norm-convergent subsequence.

Now we show that the sequence $\left(x_{n}\right)$ is almost limited; that is, for each disjoint weak* null sequence $\left(x_{n}^{*}\right)$ in $c^{*}=\ell_{1}$, where $x_{n}^{*}=\left(\lambda_{n, k}\right)_{k=1}^{\infty}$, we have $x_{n}^{*}\left(x_{n}\right) \rightarrow 0$. In fact, since $\left(y_{n}\right)$ is almost limited, $\sum_{k=1}^{\infty} \lambda_{n, k} y_{n, k} \rightarrow 0$. Also, since $\left(x_{n}^{*}\right)$ is weak ${ }^{*}$ null, for all $t=\left(t_{k}\right)_{k=1}^{\infty}$ in $c, \sum_{k=1}^{\infty} \lambda_{n, k} t_{k} \rightarrow 0$. In particular, for $t=(1,1,1, \ldots)$, $\sum_{k=1}^{\infty} \lambda_{n, k} \rightarrow 0$ as $n \rightarrow \infty$. Therefore,

$$
\begin{aligned}
x_{n}^{*}\left(x_{n}\right) & =x_{n}^{*}\left(y_{n, k}-\alpha_{n}\right) \\
& =\sum_{k=1}^{\infty} \lambda_{n, k}\left(y_{n, k}-\alpha_{n}\right) \\
& =\sum_{k=1}^{\infty} \lambda_{n, k} y_{n, k}-\sum_{k=1}^{\infty} \lambda_{n, k} \alpha_{n} \rightarrow 0 .
\end{aligned}
$$

On the other hand, the bounded sequence $\left(x_{n}\right)$ in $c_{0}$ has a weakly Cauchy subsequence. Without loss of generality, we may assume that $\left(x_{n}\right)$ is weakly Cauchy. Since the sequence $\left(x_{n}\right)$ is not norm-convergent, we can choose a sequence $n_{1}<n_{2}<\cdots$, so that $z_{1}=x_{n_{1}}-x_{n_{2}}, z_{2}=x_{n_{3}}-x_{n_{4}}, \ldots$ has no norm-convergent subsequence. This sequence of differences is almost limited and weakly null and it lies in $c_{0}$. Since $c_{0}$ has the strong GP property, the sequence $\left(z_{n}\right)$ is relatively compact and so has a norm-null subsequence, which is a contradiction.

Recall from [13] that a Banach lattice $E$ has the positive Schur property if every weakly null sequence with positive terms in $E$ is norm null. It is clear that the Schur property implies the positive Schur, but the converse is false, in general. In fact, $L^{1}[0,1]$ is a Banach lattice with the positive Schur and without the Schur property. By a remark in [14] for discrete Banach lattices, the positive Schur property and the Schur property coincide, and in the following result we show that this holds for Banach lattices with the strong GP property, too.

Theorem 2.5. For a Banach lattice E, the following are equivalent:

(a) $E$ is discrete with the positive Schur property;

(b) $E$ is a strong GP space with the positive Schur property;

(c) E has the Schur property.

Proof. (a) $\Rightarrow$ (b). Since every Banach lattice with the positive Schur property has order-continuous norm, by Theorem 2.1, $E$ is a strong GP space.

(b) $\Rightarrow(\mathrm{c})$. Let $A$ be a relatively weakly compact subset of $E$. Since $E$ has the positive Schur property, by [11, Corollary 3.6.8], $A$ is $L$-weakly compact (i.e., $\left\|x_{n}\right\| \rightarrow 0$ for every disjoint sequence $\left(x_{n}\right)$ contained in the solid hull of $A$; cf. [11, Definition 3.6.1]). By [6, Theorem 2.6], $A$ is an almost limited set, and so it 
is relatively compact, by the strong GP property of $E$. Hence $E$ has the Schur property.

$(\mathrm{c}) \Rightarrow(\mathrm{a})$. It is obvious.

From [3], a Banach space $X$ has the $\mathrm{DP}^{*}$ property whenever every relatively weakly compact set in $X$ is limited. Also, a Banach lattice $E$ has the weak DP* property if every relatively weakly compact set in $E$ is almost limited (see [6]). Clearly, every Banach lattice with the Schur property has the weak DP* property. Since every Banach lattice with order-continuous norm is a GP space, $E$ has the Schur property if and only if $E$ has both order-continuous norm and the DP* property. As a similar consequence, we have the following corollary.

Corollary 2.6. A Banach lattice $E$ has the Schur property if and only if $E$ has the strong GP property and weak DP* property.

Proof. The "only if" part follows immediately from the definition of the Schur property and Theorem 2.1.

For the "if" part, assume that $A$ is a relatively weakly compact subset of $E$. The weak $\mathrm{DP}^{*}$ property of $E$ implies that $A$ is almost limited, and so it is relatively compact, by the strong GP property. Hence, $E$ has the Schur property.

Now we have the following easy result, and we omit its proof.

Corollary 2.7. Let $E$ be a Banach lattice. Then the following are equivalent:

(a) E has the DP* and GP properties;

(b) E has the weak DP* and strong GP properties;

(c) E has the Schur property.

It is clear that every Banach lattice without the GP property, such as $\ell_{\infty}$, does not have the strong GP property. The following theorem shows that the converse is false. Also, it shows that the discreteness condition in Theorem 2.1 cannot be removed.

Theorem 2.8. Let $E$ be a nondiscrete Banach lattice with order-continuous norm. Then $E$ does not have the strong GP property.

Proof. By [1, Corollary 21.13], there exists an order interval in $E$ which is not compact. Since $E$ is $\sigma$-Dedekind complete by [6, Lemma 2.2], each order interval in $E$ is almost limited; that is, $E$ does not have the strong GP property.

As an application of the above Theorem $2.8, L^{1}[0,1]$ does not have the strong GP property, but it has the GP property.

Note that a $\sigma$-Dedekind complete Banach lattice has the GP property if and only if the norm of $E$ is order-continuous (see [5]). In the following theorem we give another proof for it.

Theorem 2.9. Let $E$ be a $\sigma$-Dedekind complete Banach lattice with the GP property. Then E has order-continuous norm.

Proof. By [8, Lemma 3.7], each order-bounded disjoint sequence in a $\sigma$-Dedekind complete Banach lattice $E$ is limited. Now, if $\left(x_{n}\right)$ is an order-bounded disjoint 
sequence in $E$ with the GP property, then by [2] the sequence $\left(x_{n}\right)$ is weakly null and limited, and so it is norm null by the GP property. So, by [2, Theorem 4.14], $E$ has order-continuous norm.

It should be noted that every order-bounded disjoint sequence in a Banach lattice is not limited or even almost limited, in general. The following examples show also that Banach lattices $c$ and $C[0,1]$ do not have the weak $\mathrm{DP}^{*}$ property.

Example 2.10. Consider the space $c$ with the sup norm. Clearly, the sequence $\left(e_{n}\right)$ of unit vectors in $c$ is order-bounded disjoint from below by 0 and from above by the vector $e=(1,1,1, \ldots)$. Define $f_{k}$ on $c$ by $f_{k}\left(x_{n}\right)=x_{k}-\lim _{n \rightarrow \infty} x_{n}$. Then $f_{k}(x) \rightarrow 0$ as $k \rightarrow \infty$ for every $x=\left(x_{n}\right) \in c$, but $\sup _{n}\left|f_{k}\left(e_{n}\right)\right| \geq f_{k}\left(e_{k}\right)=1$. We have just shown that $\left\{e_{n}: n \in N\right\}$ is not limited.

Also, the sequence $x_{n}=\left(0,0, \ldots, 0,1_{(2 n)}, 0,0, \ldots\right)$ is order-bounded disjoint in $c$, but it is not almost limited. In fact, let $g_{n} \in c^{*}=\ell_{1}(n=1,2,3, \ldots)$ be defined by $g_{n}=\left(0, \ldots, 0,1_{(2 n)},-1_{(2 n+1)}, 0, \ldots\right)$. Then $\left(g_{n}\right)$ is a disjoint weak* null sequence in $c^{*}$, but $g_{n}\left(x_{n}\right)=1$, for all $n \in N$.

Example 2.11. Let $\left(x_{n}\right)$ be a convergent sequence of distinct points in $[0,1]$. There is a bounded disjoint sequence $\left(f_{n}\right)$ in $C[0,1]$ such that $f_{n}\left(x_{2 n-1}\right)=1$ and $f_{n}\left(x_{m}\right)=0$ for all other $m$. The sequence of point functionals $\delta\left(x_{2 n-1}\right)-\delta\left(x_{2 n}\right)$ is weak* null and disjoint in $(C[0,1])^{*}$, but does not converge to 0 uniformly on $\left(f_{n}\right)$; that is, the sequence $\left(f_{n}\right)$ is not almost limited.

As we said before, from [7], a Banach space $X$ has the GP property if and only if every weakly null and limited sequence in $X$ is norm null. But if a Banach lattice $E$ has the strong GP property, then every weakly null and almost limited sequence in $E$ is norm null. The following theorem shows that the converse is false, in general.

Theorem 2.12. Let $K$ be a compact metric space. Then every weakly null and almost limited sequence in $C(K)$ is norm null. Also, if $K$ is $\sigma$-Stonian (i.e., the closure of every open $F_{\sigma}$ set is open), then $C(K)$ does not have the strong GP property.

Proof. Suppose that there is a weakly null and almost limited sequence $\left(f_{n}\right)$ in $C(K)$ which is not norm null. Then we can assume that there exists a sequence $\left(x_{n}\right)$ in $K, \epsilon>0$ such that $\left|f_{n}\left(x_{n}\right)\right| \geq \epsilon$, for all $n \in N$.

The point functionals at $\left(x_{n}\right)$ converge weak* to the point functional at 0 . Since the sequence $\left(f_{n}\right)$ is weakly null, we may assume that $f_{n}\left(x_{m}\right)$ is small if $n>m$. Use the sequence of functionals $\delta\left(x_{2 n}\right)-\delta\left(x_{2 n-1}\right)$ applied to $\left(f_{2 n}\right)$. This difference sequence of point functionals is weak ${ }^{*}$ null and disjoint but does not converge to 0 uniformly on $\left(f_{n}\right)$.

Now, assume that $K$ is $\sigma$-Stonian. We show that $C(K)$ does not have the strong GP property. In fact, let $\left(\mu_{n}\right)$ be a weak* null and disjoint sequence in dual space $C(K)^{*}$. Since $K$ is $\sigma$-Stonian, by [11, Proposition 2.1.5], $C(K)$ is $\sigma$-Dedekind complete and so by [6, Lemma 2.2], the sequence $\left(\left|\mu_{n}\right|\right)$ is weak* null. From [16], $C(K)$ has the dual positive Schur property; that is, every weak* null and positive sequence in its dual is norm null, and so the sequence $\left(\left|\mu_{n}\right|\right)$ is norm null. Hence 
$B_{C(K)}$ is an almost limited set, but it is not relatively compact; that is, $C(K)$ does not have the strong GP property.

It is well known that every limited set is conditionally weakly compact (see [4]), and the Josefson-Nissenzweig theorem precludes any possibility of the closed unit ball of an infinite-dimensional Banach space being limited. However, $B_{\ell_{\infty}}$ is indeed almost limited, and, by Rosenthal's $\ell_{1}$-theorem, $B_{\ell_{\infty}}$ is not conditionally weakly compact.

By [11], an element $e$ in a Banach lattice $E$ is called a weak unit if $B_{e}=E$, where $B_{e}$ is the band generated by $e$. For example, $C[0,1]$ is a Banach lattice with the weak unit $u(t)=t$. Banach lattices $M[0,1]$, of all signed Borel measures on $[0,1]$ of bounded variation, and $\left(\ell_{\infty}\right)^{*}$ do not have any weak unit. For the proof of the following Theorem 2.14, we remember a lemma from [11].

Lemma 2.13 ([11, Theorem 2.5.9]). Let $E$ be a Banach lattice such that $E^{*}$ has a weak unit or $E$ has order-continuous norm. Then a norm-bounded set $A$ in $E$ is conditionally weakly compact if and only if $\sup \left\{\left|f_{n}\right|(|x|): x \in A\right\} \rightarrow 0$ for every order-bounded disjoint sequence $\left(f_{n}\right)$ in $E^{*}$.

Theorem 2.14. Let $E$ be a Banach lattice such that $E^{*}$ has a weak unit or $E$ has order-continuous norm. Then every almost limited set $A$ in $E$ is conditionally weakly compact.

Proof. We need to show only that $\sup \left\{\left|f_{n}\right|(|x|): x \in A\right\} \rightarrow 0$ for every orderbounded disjoint sequence $\left(f_{n}\right)$ in $E^{*}$. Suppose that there exists some $\epsilon>0$ such that $\sup \left\{\left|f_{n}\right|(|x|): x \in A\right\}>\epsilon$ for all $n$. For each $n$, choose some $x_{n} \in A$ and some $g_{n} \in E^{*}$ with $\left|g_{n}\right| \leq\left|f_{n}\right|$ such that $\left|g_{n}\left(x_{n}\right)\right|>\epsilon$. Clearly, $\left(g_{n}\right)$ is likewise an order-bounded disjoint sequence, and, by [2], $\left(g_{n}\right)$ is weak* null. Since $A$ is almost limited, $\left(g_{n}\right)$ converges uniformly to 0 on $A$, which implies that $\left|g_{n}\left(x_{n}\right)\right| \rightarrow 0$. This leads to a contradiction.

Note that if $E$ has order-continuous norm and $A$ is an almost limited set in $E$, then not only $A$ is conditionally weakly compact, but, by [6, Theorem 2.6], $A$ is relatively weakly compact.

Theorem 2.15. Let $E$ be a Banach lattice such that $E^{*}$ has a weak unit or $E$ has order-continuous norm. Then $E$ has the strong GP property if and only if every weakly null and almost limited sequence in $E$ is norm null.

Proof. If $E$ has the strong GP property, then every weakly null and almost limited sequence in $E$ is norm null. For the converse, let $A$ be an almost limited subset of $E$. Then, by Theorem 2.14, every sequence $\left(x_{n}\right)$ in $A$ has a subsequence, denoted again by $\left(x_{n}\right)$, that is weakly Cauchy. On the other hand, the difference set $A-A$ is almost limited. Thus the sequence $\left(x_{n}-x_{m}\right)$ is almost limited and weakly null and, by the hypothesis, it is norm null. That is, the sequence $\left(x_{n}\right)$ is Cauchy and so is norm convergent in the Banach lattice $E$. Thus $A$ is relatively compact.

It should be noted that every weakly null and almost limited sequence in a Banach lattice is not norm null, in general. For example, the Rademacher functions in $L^{1}[0,1]$ are weakly null and almost limited by the weak DP* property, but they are not norm null (see [2, p. 168]). 


\section{Almost limited Completely continuous operators}

In this section we will define a new class of operators called almost limited completely continuous (alcc) operators and establish some additional properties of them. From [12], an operator $T: X \rightarrow Y$ between two Banach spaces is limited completely continuous if $T$ carries weakly null and limited sequences in $X$ to norm null ones, and the class of lcc operators is denoted by $\operatorname{Lcc}(X, Y)$.

Definition 3.1. A bounded linear operator $T$ from a Banach lattice $E$ into a Banach space $Y$ is alcc if it carries weakly null and almost limited sequences in $E$ to norm null ones. We denote this class of operators by $L^{a} c c(E, Y)$.

It is easy to see that the operator space $L^{a} c c(E, Y)$ is a norm-closed subspace of $L(E, Y)$. By [12, Theorem 2.8], a Banach space $X$ has the $\mathrm{DP}^{*}$ property if and only if, for each Banach space $Y, C c(X, Y)=\operatorname{Lcc}(X, Y)$, where $C c(X, Y)$ is the class of completely continuous operators. If a Banach lattice $E$ has the weak DP* property, then, for every Banach space $Y, L^{a} c c(E, Y)=C c(E, Y)$. It is clear that if a Banach lattice $E$ has the $\mathrm{DP}^{*}$ property, then, for every Banach space $Y$, $L^{a} c c(E, Y)=\operatorname{Lcc}(E, Y)=C c(E, Y)$.

Theorem 3.2. A bounded linear operator $T$ on $E$ is an alcc operator if, for each almost limited set $A \subseteq E$, the set $T(A)$ is relatively compact.

Proof. If $\left(x_{n}\right)$ is a weakly null and almost limited sequence in $E$, then it is enough to show that every subsequence of $\left(x_{n}\right)$ has a subsequence $\left(x_{n_{k}}\right)$ such that $\left(T x_{n_{k}}\right)$ is norm null.

For each such subsequence of $\left(x_{n}\right)$, which is denoted again by $\left(x_{n}\right)$, by hypothesis, the sequence $\left(T x_{n}\right)$ is relatively compact, and so it has a convergent subsequence $\left(T x_{n_{k}}\right)$. Since $T$ carries weakly null sequences to weakly null ones, the sequence $\left(T x_{n_{k}}\right)$ is weakly null, and so $\left\|T x_{n_{k}}\right\| \rightarrow 0$ as $k \rightarrow \infty$. This shows that the sequence $\left(T x_{n}\right)$ is norm null, and then $T$ is alcc.

It should be noted that the converse of the theorem is false, in general. In fact, let $K$ be a compact Hausdorff space. Then, by Theorem 2.12, every weakly null and almost limited sequence in $C(K)$ is norm null and so the identity operator $\operatorname{Id}_{C(K)}$ is alcc, but, if $K$ is $\sigma$-Stonian, then there exists an almost limited set $A$ in $C(K)$ such that $A$ is not relatively compact.

Theorem 3.3. Let $T: E \rightarrow Y$ be an alcc operator from a Banach lattice $E$ to a Banach space $Y$ such that $E^{*}$ has a weak unit or $E$ has order-continuous norm. Then, for every almost limited set $A$ in $E, T(A)$ is relatively compact.

Proof. Let $A \subseteq E$ be almost limited. Then, by Theorem 2.14, every sequence $\left(x_{n}\right)$ in $A$ has a subsequence, denoted again by $\left(x_{n}\right)$, that is weakly Cauchy. On the other hand, the difference set $A-A$ is almost limited. So the sequence $\left(x_{n}-x_{m}\right)$ is weakly null and almost limited, and then the sequence $\left(T x_{n}\right)$ is Cauchy and so it is norm convergent in the Banach space $Y$. Thus $T(A)$ is relatively compact.

In the following theorem, we give a characterization of the strong GP property, with respect to alcc operators. 
Theorem 3.4. For a Banach lattice E such that $E^{*}$ has a weak unit or E has order-continuous norm, the following are equivalent:

(a) E has the strong GP property;

(b) for each Banach space $Y, L^{a} c c(E, Y)=L(E, Y)$;

(c) $L^{a} c c\left(E, \ell_{\infty}\right)=L\left(E, \ell_{\infty}\right)$.

Proof. (a) $\Rightarrow$ (b). Let $T: E \rightarrow Y$, and let $A$ be an almost limited subset of $E$. Then $A$ as well as $T(A)$ are relatively compact, since $E$ has the strong GP property. Hence, by Theorem 3.2, $T$ is an alcc operator.

(b) $\Rightarrow$ (c). It is obvious.

(c) $\Rightarrow$ (a). If $E$ does not have the strong GP property, then, by Theorem 2.15, there exists a weakly null and almost limited sequence $\left(x_{n}\right)$ in $E$ such that $\left\|x_{n}\right\|=$ 1 , for all $n$. Choose a normalized sequence $\left(x_{n}^{*}\right)$ in $E^{*}$ such that $\left|\left\langle x_{n}, x_{n}^{*}\right\rangle\right|=1$, for all $n$, and define the operator $T: E \rightarrow \ell_{\infty}$ by

$$
T x=\left(\left\langle x, x_{n}^{*}\right\rangle\right), \quad x \in E .
$$

But $T$ is not alcc, since the sequence $\left(x_{n}\right)$ is weakly null and almost limited and $\left\|T x_{n}\right\| \geq 1$, for all $n$.

We note that if $E^{*}$ does not have a weak unit and $E$ does not have ordercontinuous norm, then Theorem 3.4 may be false. In fact, by Theorem 2.12, every weakly null and almost limited sequence in $C(K)$, where $K$ is a compact Hausdorff space, is norm null and so every operator on $C(K)$ is alcc, but $C(K)$ does not have the strong GP property.

Corollary 3.5. Every bounded linear operator on a discrete Banach lattice $E$ with order-continuous norm is an alcc operator.

Proof. By Theorem 2.1, E has the strong GP property.

It is clear that, if $\mathcal{M} \subset L(X, Y)$ is a Banach lattice with the strong GP property, then all of the evaluation operators $\phi_{x}$ and $\psi_{y^{*}}$ are alcc operators, where $\phi_{x}(T)=$ $T x$ and $\psi_{y^{*}}(T)=T^{*} y^{*}$, for $x \in X, y^{*} \in Y^{*}$, and $T \in \mathcal{M}$. Now, we show that the alccness of evaluation operators is a sufficient condition for the strong GP property of their domain.

Theorem 3.6. Assume that $Y$ has the Schur property and that $\mathcal{M} \subset L(X, Y)$ is a Banach lattice such that $\mathcal{M}^{*}$ has a weak unit or $\mathcal{M}$ has order-continuous norm. If, for every $y^{*} \in Y^{*}$, the evaluation operator $\psi_{y^{*}}$ on $\mathcal{M}$ is alcc, then $\mathcal{M}$ has the strong GP property.

Proof. If $\mathcal{M}$ does not have the strong GP property, then, by Theorem 2.15, there exist a weakly null and almost limited sequence $\left(T_{n}\right)$ in $\mathcal{M}$ and some $\epsilon>0$ such that $\left\|T_{n}\right\|>\epsilon$, for all $n$. So there exists a sequence $\left(x_{n}\right)$ in $B_{X}$ such that $\left\|T_{n}\left(x_{n}\right)\right\|>\epsilon$, for all $n$. On the other hand, the evaluation operator $\psi_{y^{*}}$ on $\mathcal{M}$ is alcc for all $y^{*} \in Y^{*}$ and so $\left\|T_{n}^{*}\left(y^{*}\right)\right\|=\left\|\psi_{y^{*}}\left(T_{n}\right)\right\| \rightarrow 0$. Hence $\left|\left\langle T_{n} x_{n}, y^{*}\right\rangle\right| \leq$ $\left\|T_{n}^{*}\left(y^{*}\right)\right\| \rightarrow 0$. So the sequence $\left(T_{n} x_{n}\right)$ is weakly null and it is norm null by the Schur property, a fact that is impossible. 
Theorem 3.7. Assume that $X$ has the Schur property and that $\mathcal{M} \subset L_{w^{*}}\left(X^{*}, Y\right)$ is a Banach lattice such that $\mathcal{M}^{*}$ has a weak unit or $\mathcal{M}$ has order-continuous norm. If, for every $x^{*} \in X^{*}$, the evaluation operator $\phi_{x^{*}}$ on $\mathcal{M}$ is alcc, then $\mathcal{M}$ has the strong GP property.

Proof. If $\mathcal{M}$ does not have the strong GP property, then, by Theorem 2.15, there exist a weakly null and almost limited sequence $\left(T_{n}\right)$ in $\mathcal{M}$ and some $\epsilon>0$ such that $\left\|T_{n}\right\|>\epsilon$, for all $n$. On the other hand, the evaluation operator $\phi_{x^{*}}$ on $\mathcal{M}$ is alcc for all $x^{*} \in X^{*}$ and so $\left\|T_{n}\left(x^{*}\right)\right\|=\left\|\phi_{x^{*}}\left(T_{n}\right)\right\| \rightarrow 0$. Since $\left\|T_{n}^{*}\right\|>\epsilon$, there exists a sequence $\left(y_{n}^{*}\right)$ in $B_{Y^{*}}$ such that $\left\|T_{n}^{*} y_{n}^{*}\right\|>\epsilon$, for all $n$. But the Schur property of $X$ shows that the weakly null sequence $\left(T_{n}^{*} y_{n}^{*}\right)$ is norm null, which is a contradiction.

Note that continuous linear images of limited sets or sequences are limited, but the same conclusion is false for almost limited sets or sequences, in general. So in Theorem 3.4, one cannot replace the strong GP property of the domain $E$ of related operators, by their images. In fact, the Banach lattice $c_{0}$ has the strong GP property, but the operators $T: L^{1}[0,2 \pi] \rightarrow c_{0}$ and $S: L^{1}[0,1] \rightarrow c_{0}$ defined by

$$
\begin{aligned}
& T f=\left(\int_{0}^{2 \pi} f(t) \sin (n t) d t\right) \quad \text { for all } f \in L^{1}[0,2 \pi], \\
& S g=\left(\int_{0}^{1} g(t) r_{n}(t) d t\right) \quad \text { for all } g \in L^{1}[0,1]
\end{aligned}
$$

are not alcc operators since the sequences $f_{n}(t)=\sin (n t)$ and $g_{n}(t)=r_{n}(t)$ are weakly null and almost limited; but $\left\|T f_{n}\right\|=\pi$ and $\left\|S g_{n}\right\|=1$, for all $n$. Note that the sequences $\left(T f_{n}\right)$ and $\left(S g_{n}\right)$ are not almost limited.

In the following, we establish some conditions which guarantee that the continuous linear images of almost limited sets are also almost limited. Recall from [11] that a positive linear operator $T: E \rightarrow F$ between two Banach lattices is almost interval-preserving, if $T[0, x]$ is dense in $[0, T x]$, for every $x \in E^{+}$.

Theorem 3.8. Let $T: E \rightarrow F$ between two Banach lattices be an almost intervalpreserving operator, and let $A$ be an almost limited subset of $E$. Then $T(A)$ is almost limited in $F$.

Proof. Let $\left(y_{n}^{*}\right)$ be a disjoint weak* null sequence in $F^{*}$. By [11, Theorem 1.4.19], $T^{*}$ is a lattice homomorphism and so $\left(T^{*} y_{n}^{*}\right)$ is a disjoint weak* null sequence in $E^{*}$. Since $A$ is almost limited,

$$
\limsup _{x \in A}\left|\left\langle T x, y_{n}^{*}\right\rangle\right|=\limsup _{x \in A}\left|\left\langle x, T^{*} y_{n}^{*}\right\rangle\right| \rightarrow 0 .
$$

This completes the proof.

Corollary 3.9. If a Banach lattice $F$ has the strong GP property, then every almost interval-preserving operator $T: E \rightarrow F$ is alcc.

Proof. Let $A$ be an almost limited set in $E$, and let $T: E \rightarrow F$ be an almost interval-preserving operator. Then, by Theorem $3.8, T(A)$ is almost limited and so 
it is relatively compact, by the strong GP property of $F$. Hence, by Theorem 3.3, $T$ is alcc.

From [2], an operator $T$ from a Banach lattice $E$ into another $F$ is said to be order-bounded if, for each $x \in E^{+}$, the subset $T([-x, x])$ is order-bounded in $F$.

Theorem 3.10. Let $E$ and $F$ be two Banach lattices such that $E^{*}$ has the weak* sequentially continuous lattice operations or $F$ is $\sigma$-Dedekind complete. If the operator $T: E \rightarrow F$ is order-bounded, then $T(A)$ is an almost limited set in $F$, whenever $A$ is an almost limited solid set in $E$.

Proof. If $E^{*}$ has the weak ${ }^{*}$ sequentially continuous lattice operations and $A$ is an almost limited solid set in $E$, then, by [10, Theorem 2.6], $A$ is limited. So $T(A)$ is limited.

If $F$ is $\sigma$-Dedekind complete and $\left(f_{n}\right)$ is a disjoint weak ${ }^{*}$ null sequence in $F^{*}$, then, by [6, Lemma 2.2] and [10, Theorem 2.7], the sequences $\left(\left|f_{n}\right|\right)$ and $\left(\left|T^{*}\left(f_{n}\right)\right|\right)$ are weak* null. Now, to finish the proof, we have to show that $\sup _{y \in T A}\left|f_{n}(y)\right|=$ $\sup _{x \in A}\left|T^{*} f_{n}(x)\right| \rightarrow 0$. Let $\left(x_{n}\right) \subseteq A^{+}$be a disjoint sequence. Since the sequence $\left(\left|T^{*}\left(f_{n}\right)\right|\right)$ is weak* null in $E^{*}$, by [2, p. 77], there exists a disjoint sequence $\left(g_{n}\right) \subseteq E^{*}$ such that $\left|g_{n}\right| \leq\left|T^{*} f_{n}\right|$ and $g_{n}\left(x_{n}\right)=\left(T^{*} f_{n}\right)\left(x_{n}\right)$, for all $n$. It is easy to see that the sequence $\left(g_{n}\right)$ is weak* null. As the set $A$ is almost limited, $\sqrt[n]{\sup _{x \in A}}\left|g_{n}(x)\right| \rightarrow 0$. From the inequality

$$
\left|T^{*} f_{n}\left(x_{n}\right)\right|=\left|g_{n}\left(x_{n}\right)\right| \leq \sup _{x \in A}\left|g_{n}(x)\right|,
$$

we conclude that $T^{*} f_{n}\left(x_{n}\right) \rightarrow 0$. Now, by [10, Theorem 2.7], we have

$$
\sup _{x \in A}\left|T^{*} f_{n}\right|(|x|)=\sup _{x \in A}\left|T^{*} f_{n}\right|(x) \rightarrow 0 .
$$

Thus by the inequality $\left|T^{*} f_{n}(x)\right| \leq\left|T^{*} f_{n}\right|(|x|)$, we see that $\sup _{x \in A}\left|T^{*} f_{n}(x)\right| \rightarrow 0$, and so $T(A)$ is almost limited.

Theorem 3.11. Let $T: E \rightarrow F$ and $S: F \rightarrow G$ be almost interval-preserving operators such that $S$ or $T$ is alcc. Then the composition operator $S T$ is alcc.

Proof. Suppose that $S$ is alcc, and let $\left(x_{n}\right)$ be a weakly null and almost limited sequence in $E$. Then the sequence $\left(T x_{n}\right)$ is weakly null and, by Theorem 3.8, it is almost limited. Since $S$ is an alcc operator, $\left\|S T\left(x_{n}\right)\right\| \rightarrow 0$, and so the composition operator $S T$ is alcc. If $T$ is alcc, then clearly the operator $S T$ is alcc.

Theorem 3.12. Let $T: X \rightarrow E$ be a bounded linear operator, and assume that $E^{*}$ has a weak unit or that $E$ has order-continuous norm. Then the implications $(a) \Rightarrow(b) \Rightarrow(c)$ are valid for the following assertions:

(a) $T$ is almost limited; that is, $T\left(B_{X}\right)$ is almost limited in $E$;

(b) for each Banach space $Y$ and each alcc operator $S: E \rightarrow Y$, the composition operator $S T$ is compact;

(c) for each almost interval-preserving operator $S: E \rightarrow F$, where $F$ has the strong GP property, the operator ST is compact. 
Proof. (a) $\Rightarrow$ (b). If $T$ is almost limited, then $T\left(B_{X}\right)$ is almost limited in $E$. Since $S: E \rightarrow Y$ is alcc, by Theorem 3.3, $S\left(T\left(B_{X}\right)\right)$ is relatively compact. So the operator $S T$ is compact.

(b) $\Rightarrow$ (c). It follows from Corollary 3.9.

\section{REFERENCES}

1. C. D. Aliprantis and O. Burkishaw, Locally Solid Riesz Spaces, Pure Appl. Math. 76, Academic Press, New York, 1978. MR0493242. 16, 17, 19

2. C. D. Aliprantis and O. Burkishaw, Positive Operators, Academic Press, New York, 1978. $16,20,21,25$

3. J. Borwein, M. Fabian, and J. Vanderwerff, Characterizations of Banach spaces via convex and other locally Lipschitz functions, Acta Math. Vietnam 22 (1997), no. 1, 53-69. Zbl 0920.46004. MR1479738. 19

4. J. Bourgain and J. Diestel, Limited operators and strict cosingularity, Math. Nachr. 119 (1984), 55-58. Zbl 0601.47019. MR0774176. DOI 10.1002/mana.19841190105. 16, 21

5. A. V. Buhvalov, Locally convex spaces that are generated by weakly compact sets (in Russian), Vestnik Leningrad Univ. Mat. Meh. Astronom. 7 (1973), 11-17. MR0361701. 19

6. J. X. Chen, Z. L.Chen, and G. X. Ji, Almost limited sets in Banach lattices, J. Math. Anal. Appl. 412 (2014), no. 1, 547-563. Zbl pre06420079. MR3145821. DOI 10.1016/j.jmaa.2013.10.085. 15, 17, 18, 19, 20, 21, 25

7. L. Drewnowski, On Banach spaces with the Gelfand-Phillips property, Math. Z. 193 (1986), no. 3, 405-411. Zbl 0629.46020. MR0862887. DOI 10.1007/BF01229808. 16, 20

8. A. El Kaddouri and M. Moussa, About the class of ordered limited operators, Acta Univ. Carolin. Math. Phys. 54 (2013), no. 1, 37-43. Zbl 1307.46008. MR3222749. 19

9. G. Emmanuele, On Banach spaces with the Gelfand-Phillips property, III, J. Math. Pures Appl. (9) 72 (1993), no. 3, 327-333. Zbl 0830.46011. MR1225513. 16

10. N. Machrafi, A. Elbour, and M. Moussa, Some characterizations of almost limited sets and applications, preprint, arXiv:1312.2770v1 [math.FA]. 17, 25

11. P. Meyer-Nieberg, Banach Lattices, Springer, Berlin, 1991. MR1128093. DOI 10.1007/978-3-642-76724-1. 16, 17, 18, 20, 21, 24

12. M. Salimi and S. M. Moshtaghioun, The Gelfand-Phillips property in closed subspaces of some operator spaces, Banach J. Math. Anal. 5 (2011), no. 2, 84-92. MR2792501. DOI 10.15352/bjma/1313363004. 22

13. J. A. Sánchez Henríquez, The positive Schur property in Banach lattices, Extracta Math. 7, (1992), no. 2-3, 161-163. MR1248468. 18

14. W. Wnuk, A note on the positive Schur property, Glasgow Math. J. 31 (1989), no. 2, 169-172. Zbl 0694.46020. MR0997812. DOI 10.1017/S0017089500007692. 18

15. W. Wnuk, Banach Lattices with Order Continuous Norms, Polish Scientific Publishers PWN, Warsaw, 1999. 17

16. W. Wnuk, On the dual positive Schur property in Banach lattices, Positivity 17 (2013), no. 3, 759-773. Zbl pre06209115. MR3090691. DOI 10.1007/s11117-012-0203-7. 20

Department of Mathematics, University of Yazd, Yazd, Iran.

E-mail address: halimeh_ardakani@yahoo.com

E-mail address: moshtagh@yazd.ac.ir

E-mail address: smodarres@yazd.ac.ir

E-mail address: m_salimi52@yahoo.com 